\title{
Using artificial neural networks for GNSS observations analysis and displacement prediction of suspension highway bridge
}

\author{
Ashraf A. A. Beshr ${ }^{1}$ (1) Fawzi H. Zarzoura ${ }^{1}$ \\ Received: 26 November 2020 / Accepted: 7 January 2021 / Published online: 3 March 2021 \\ (c) The Author(s) 2021
}

\begin{abstract}
Bridges are playing a major role in the socio-economic development of any country over the world. Suspension highway bridges are one of the most sensitive structures to various external influences and loads. Therefore, the need for structural monitoring system, maintenance, and deformation prediction for these structures is important and vital. One of the main objectives of monitoring the structural deformation is predicting the deformation values, which will help to avoid sudden failure and accidents in the future. Artificial neural networks (ANNs) and adaptive neuro-fuzzy inference systems (ANFIS) have proven successful solution in many engineering applications and problems. This paper investigates an integrated monitoring system using GNSS observations for studying the deformation behavior and displacement prediction for suspension highway bridge, taking into consideration the effect of wind, temperature, humidity and traffic loads during the operational and short-term measurements. Due to the complexity of the mathematical processing of large GNSS monitoring data for obtaining reliable results, adequate model of several alternatives should be chosen. One of the main objectives of this paper is to investigate the optimum predictive model for analysis of GNSS observations and displacement prediction. Several models are applied and compared for prediction of suspension bridge displacement for both kinematic and dynamic models. The resulting predicted displacement values by applying artificial neural networks (ANNs) and ANFIS provide a significant improvement for predicting the structure deformation values for suspension highway bridges from GNSS observations.
\end{abstract}

Keywords GNSS $\cdot$ Prediction $\cdot$ Suspension bridges $\cdot$ Monitoring $\cdot$ Artificial neural networks (ANNs)

\section{Introduction}

Automatic monitoring of structures is the process of automated day and night observations and recording all the necessary deformation parameters. The purpose of structure monitoring process is to prevent emergencies and damage or destruction of structures. Prediction (forecasting) of structural deformation helps to track any expected changes of the structure and its individual elements in the near future, which makes it possible to prevent the occurrence of negative events [1,2]. Deformation of engineering structures is divided into slow and fast movements based on the variation of time scale [3]. Deformation of bridges comes almost from

Ashraf A. A. Beshr

aaabeshr@mans.edu.eg

Fawzi H. Zarzoura

Fawzihamed@mans.edu.eg

1 Public Works Department, Faculty of Engineering, Mansoura University, Mansoura, Egypt the heat and compressive load, constant stress, strong wind load and seismic action of vehicle loads ... etc. Deformation can be defined by distance or displacement points, angular displacement and stress conditions [4].

Suspension bridges are important structure and widely used, especially in regional and urban infrastructure for various transport types due to the speed of its construction and cost [5]. One component of the safety system of these bridges can be an automatic geodetic monitoring system using global navigation satellite systems (GNSS) observations technology. Nowadays, GNSS have become an effective and valuable geodetic observations methods due to its continuous operation in real time [6]. Differential GNSS (DGNSS) is a method which can be applied to reduce or eliminate the influence of the ionosphere, the troposphere, and errors in the orbit. In addition, satellite clock error or receiver error may be eliminated by calculating the difference between the two receivers, or two satellites, respectively. Displacement of any bridge depends not only on the time but also on the impact of traffic volume and wind on 
natural-technical system of cable-stayed bridge. This complicates the task of building a predictive model $[5,7]$.

Artificial neural networks (ANNs) and adaptive neuro fuzzy inference system (ANFIS) have been applied in several engineering applications by several researchers [8-10]. ANNs technique is composed of multiple nodes which imitates biological neurons in the human brain. The neurons connect to each other through links. The ANFIS is a kind of ANN that is based on Takagi-Sugeno fuzzy inference system [10]. Researchers have successfully used ANFIS and ANN techniques in different fields of science.

Deformation of any structure can be determined by the displacement of several monitoring points fixed and distributed on the structure itself. Let the vector position of point $P$ in $3 \mathrm{D}$ coordinate system $\left(X_{\mathrm{P}}, Y_{\mathrm{P}}, Z_{\mathrm{P}}\right)$ before and after occurrence of the deformation equal to $r_{\mathrm{p}}$ and $r_{\mathrm{p}}^{\prime}$, respectively. Then vector $r_{\mathrm{p}}^{J}$ may be expressed as [11]:

$r_{\mathrm{P}}^{\prime}=f\left(x_{\mathrm{p}}, y_{\mathrm{p}}, z_{\mathrm{p}}, t\right)$.

where: $t=$ time variation between two observations epochs.

From Eq. (1), the position of any monitoring point on the monitored structure depends mainly on their initial position and time. Therefore, the displacement vector $d_{\mathrm{p}}$ for any monitoring point $\mathrm{P}$ can be calculated as following:

$d_{\mathrm{P}}=r_{\mathrm{P}}^{\prime}-r_{\mathrm{P}}=f\left(\left(x_{\mathrm{P}}-x_{0}\right),\left(y_{\mathrm{P}}-y_{0}\right),\left(z_{\mathrm{P}}-z_{0}\right),\left(t-t_{0}\right)\right)$

A prediction or forecasting is a statement about a future event. A prediction model is a study which provides information on the possible states of the monitored object in the future, or how and when to implement them. Predictive modeling uses statistics to predict outcomes. Prediction methods are based on mathematical extrapolation, in which the choice of the approximating function is a subject of the conditions and limitations of object prediction.

The prediction methods can be classified according to the prediction time [3]:

(1) Operational prediction-The prediction period with anticipation to 1 month.

(2) Short-term prediction-The prediction period with anticipation from 1 month to 1 year.

(3) Medium-term prediction-The prediction period with anticipation from 1 to 5 years.

(4) Long-term prediction-The prediction period with anticipation from 5 to 15 years.

The conditional correlation function is used in the form of predictive models to find the best predictions for prediction process and thus predicting accuracy of forecasting. The average error of approximation can be calculated as follows: $\bar{\varepsilon}=\frac{1}{n} \cdot \sum \frac{\left|y_{i}-y_{x}\right|}{y_{i}} \cdot 100$

where: $y_{i}$-empirical values of resultant variable; $y_{x}$ - the theoretical values of resultant variable.

Several researchers have used several and different mathematical algorithmic and techniques for structural monitoring in both static and kinematic mode [1, 7, 12]. For analysis and interpretation of structural deformation of the studied suspension bridge, two deformation models have been applied:

(a) Kinematic model

This model compares the geometrical properties of the monitored structure between two (or more) time intervals. This model takes into account the time factor only indirectly or based on predetermined or intended function of time (speed, acceleration, etc.).

\section{(b) Dynamic model}

In this model, deformation as the output signal, is a function of time and loads and immediately react to changes in causal forces. In addition to the kinematic model, the relationship between deformation and affecting factors are also taken into consideration.

\section{The objectives of this paper are}

(1) To investigate the capability of using ANN and ANFIS in GNSS observations analysis and displacement prediction for suspension highway bridge in kinematic and dynamic models.

(2) Several models are applied for modeling GNSS observations and predicting the displacement values of suspension bridges. Comparisons between the applied models were carried out to investigate the optimum predictive model.

(3) Study the effect of the amount of data observed and confidence limit on the applied models and techniques for GNSS observations processing and displacement prediction for suspension bridges.

\section{Applied mathematical models used for analysis of GNSS observations and displacement prediction}

In this section, several models and functions are presented to analyze GNSS observations and predict the values of displacements (in $X$ and $Y$ directions) for monitoring stations fixed on the studied suspension highway bridge.

(1) Regression models 
Regression analysis is a set of statistical processes for estimating relationships among variables. It includes several techniques and functions for modeling and analyzing multiple variables, when the focus is on the relationship between a dependent variable and one or more independent variables. Regression analysis helps to understand how the typical value of the dependent variable changes when any one of the independent variables change, while the other independent variables are fixed. The regression function is defined in terms of a finite number of unknown parameters that are estimated from the data. Several models for carrying out regression analysis have been applied in this paper.

(a) Types of regression models applied for kinematic solution:

In all kinematic models, the dependent variable is the displacement and independent variable is the observations time $(t)$. The applied regression models in this paper are Linear, where: $F$-load vehicles on the bridge (ton); $W$-wind speed $(\mathrm{m} / \mathrm{s}) ; T$-temperature $\left({ }^{\circ} \mathrm{C}\right) ; H-\Delta$ humidity $(\%), X$-displacement (or $Y$-displacement).

Each presented regression model for dynamic deformation prediction (Eqs. 4 and 5) has one or more unknown parameters of the approximating model $\left(a_{1}, a_{2}, a_{3}, \ldots, a_{n}\right)$ which must be accurately determined. This statement of the problem requires an optimization procedure in the sense of some criterion selected utility. This can be a set of parameter estimates of observed items and options for their refinement. Many mathematical functions can be applied to support a diverse set of parameters (coefficients). This is an unavoidable problem of choosing the most appropriate mathematical model based on experimental data:

$f(x)=a_{0}+a_{1} x+a_{2} x^{2}+a_{3} x^{2}+\cdots+a_{j} x^{j}=a_{0}+\sum_{k=1}^{j} a_{k} x^{k}$

The general expression for any errors using the method of least squares can be written as follow:

$$
A=\left[\begin{array}{ccccc}
n & \sum x_{i} & \sum x_{i}^{2} & \cdots & \sum x_{i}^{j} \\
\sum x_{i} & \sum x_{i}^{2} & \sum x_{i}^{3} & \cdots & \sum x_{i}^{j+1} \\
\sum x_{i}^{2} & \sum x_{i}^{3} & \sum x_{i}^{4} & \cdots & \sum x_{i}^{j+2} \\
\vdots & \vdots & \vdots & \ddots & \vdots \\
\sum x_{i}^{j} & \sum x_{i}^{j+1} & \sum x_{i}^{j+2} & \cdots & \sum x_{i}^{j+j}
\end{array}\right], \quad X=\left[\begin{array}{c}
a_{0} \\
a_{1} \\
a_{2} \\
\vdots \\
a_{j}
\end{array}\right], \quad B=\left[\begin{array}{c}
\sum y_{i} \\
\sum\left(x_{i} y_{i}\right) \\
\sum\left(x_{i}^{2} y_{i}\right) \\
\vdots \\
\sum\left(x_{i}^{j} y_{i}\right)
\end{array}\right]
$$

Logarithmic, Inverse, Quadratic, Cubic, Power, Compound, S-curve, Logistic, Growth and Exponential functions.

(b) Types of regression models used for dynamic solution:

Two multiple regression models were applied for dynamic solution. The purpose of multiple regression is to build a model with a large number of factors such as wind, temperature, humidity and traffic loads, determining at the same time and taking into consideration the influence of each factor of them separately, as well as their cumulative impacts on the simulated index. The two applied multiple regression functions are:

(i) The first assumed model is:

$$
X_{\operatorname{Reg}_{1}}(H, F, T, W, X)=a_{1}+a_{2} F+a_{3} W+a_{4} T+a_{5} H
$$

(ii) The second used model is:

$$
\begin{gathered}
X_{\mathrm{Reg} 2}(H, F, T, W, X)=a_{1}+a_{2} F+a_{3} W+a_{4} T+a_{5} H \\
\quad+a_{6} F W+a_{7} F T+a_{8} F H+a_{9} W T+a_{10} W H \\
\quad+a_{11} H T+a 12 F^{2}+a_{13} W^{2}+a_{14} T^{2}+a_{15} H^{2}
\end{gathered}
$$

There are many different approaches to solve the matrices in Eq. (7) based on different mathematical theories and methods. Therefore, the least squares adjustment technique should be applied to determine the parameters of each model and its accuracy using the designed software programming written by MATLAB language.

\section{Artificial neural networks}

A neural network is a set of elements connected in some way so that provides interaction between them. These elements, referred to as neurons or nodes that represent simple processors, have a certain combination rule input and rule activation allowing calculating the output signals for the aggregate input signals. The output signal of the other elements may be sent on weighted connections, which is associated with weighting factor or weight. Depending on the value of the weight coefficient, the transmission signal is amplified or attenuated [13, 14].

Thus, the operation of a neuron can be described by Eq. 8 [15]: 
$s_{i}=\sum_{k=1}^{n} w_{i k} x_{i}+w_{i 0} x_{0}=\sum_{j=0}^{n} w_{i j} x_{i}$

where $x_{i}$-neuron input signals; $w_{i, j}$-weights; $s_{i}$-sum input effects on neuron activation function; $x_{0}$ and $w_{0}$ - (it is also often called offset coefficient) in each artificial neural introduced specifically for the network initialization; usually $x_{0}=+1$; all coefficients $w_{0}$, like the rest of $w_{j}(j=1, \ldots, n)$ are set in the learning process Fig. 1.

ANNs are characterized by their architecture: the structure of connections between neurons, the number of layers, the activation function of neurons, learning algorithms. From this point of view can be listed as follows [15]:

- Static networks;

- Dynamic networks;

- Fuzzy neural network;

- Network self-organization.

The most widely used multi-static neural networks without feedback ( $80 \%$ of all applications of neural networks). Artificial neural networks (ANNs) have been applied for several engineering problems, including deformation prediction and forecasting over traditional statistical methods where ANNs do not require complex constitutive models [14].

\section{Adaptive neuro-fuzzy inference systems (ANFIS) based prediction}

This method is based on systems combining fuzzy logic and neural networks. The simple forms of the artificial neural networks (ANNs) are not able to give any explicit knowledge or causal relationships for a system [10, 15]. Roger [16] developed ANFIS technique that could overcome the shortcomings of ANNs and fuzzy systems.
The fuzzy part of the ANFIS is constructed by means of input and output variables, membership functions, fuzzy rules and inference method. The training inputs are also called energy drivers and are variables that can affect the output, such as, in the case study: the effect of wind, temperature, humidity and traffic loads. The membership functions of the system are the functions that define the fuzzy sets. The fuzzy rules have a form of if-then rule and define how the output must be for a specific value of membership of its inputs. In general, the fuzzy systems have different kind of inference methods but ANFIS is based on a particular type of fuzzy system with Takagi-Sugeno rules as the inference method. Figure 2 shows the architecture of an ANFIS structure with two inputs, four if-else rules and one output [0].

\section{Observations site and methodology}

Huangpu Bridge was the third longest suspension bridge in China. Construction of the bridge began in December

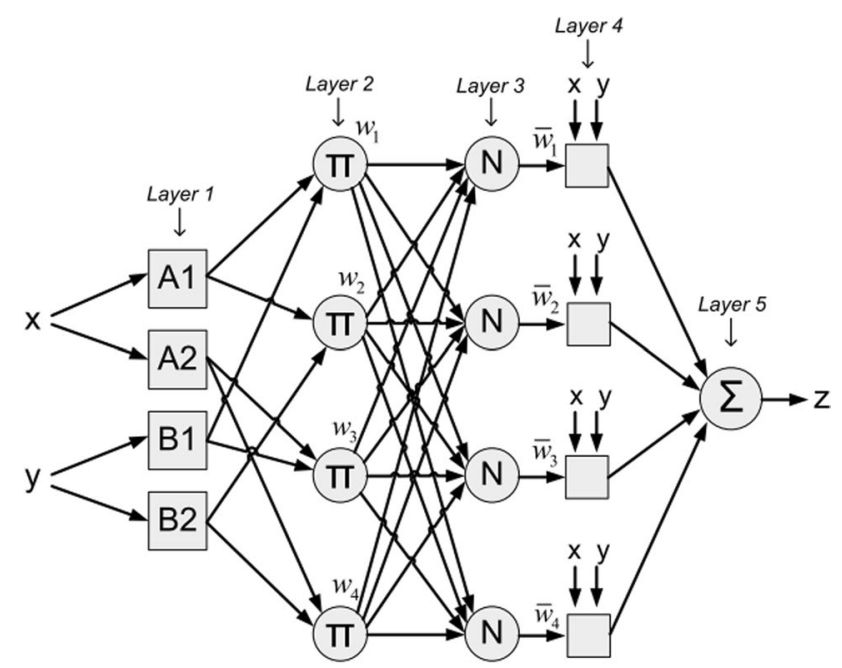

Fig. 2 ANFIS architecture with two inputs, four rules and one output
Fig. 1 Artificial neural network process

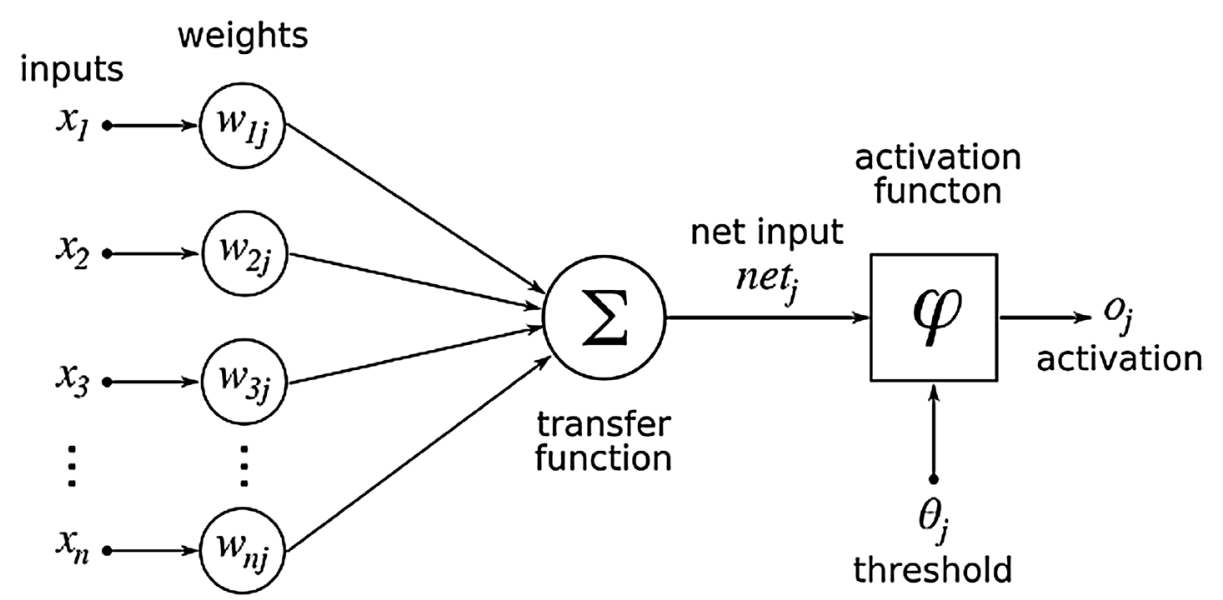


2003 and it was opened for traffic in December 2008. The bridge has a total length of $2270 \mathrm{~m}$. On the south bank of the Huangpu River, the bridge is a suspension bridge with two towers. The main passage $1108 \mathrm{~m}$ is suspended from the supports of the two main cables, while the spans are supported by the bottom side pillars. On the north bank of the Huangpu River Bridge is a cable-stayed bridge with main span of $383 \mathrm{~m}$. The opposite side near the suspension bridge has a length of $197 \mathrm{~m}$. The height of the main pylon cablestayed bridge is $201 \mathrm{~m}$ [5]. The base station is $1.00 \mathrm{~km}$ away from point 107 , and $1.00 \mathrm{~km}$ away from point $101 \mathrm{Fig}$. 3 .

Data applied in this paper was collected continuously for nearly $72 \mathrm{~h}$. For all that time period, the bridge has 13 GNSS receivers, and one base station was on the bank, Fig. 3. GNSS receivers collected information with a data rate of $10 \mathrm{~Hz}$ or higher. In addition, meteorological measurements were performed near the location of the GNSS receiver west. Operating air temperature, pressure, relative humidity and wind velocity and direction were observed every $15 \mathrm{~s}$.

\section{Solution steps}

A program using MATLAB was designed and used to calculate the coefficients of the equation. Figure 4 shows a flowchart diagram explaining the steps of applied solution for finding the parameters of the approximating model, for any argument with the monitoring time.

\section{Results and analysis}

In this paper, data applied (GNSS observations) were collected continuously for nearly $72 \mathrm{~h}$. Several mathematical models for observations analysis and displacement prediction and two cases of data amount for dynamic and kinematic state are applied and compared with confidence interval with a probability $\rho=0.95, \Delta= \pm 2 \sigma$. Several trials were done as following:

First trial: Taking $66.67 \%$ of all available data to analyze the GNSS observations and build up the prediction model and the remaining $33.33 \%$ of data for checking the model with the confidence interval with a probability $\rho=0.95$, $\Delta= \pm 2 \sigma$.
Figures 5 and 6 show samples of the results of modeling GNSS observations in the $X$-direction for one receiver station using $66.67 \%$ of all available data where the $X$-axis expresses the observations time in minutes and the $Y$-axis expresses the values of $X$ coordinates component and the red line or curve represents the modified model.

(1) Solution using kinematic models

(2) Solution using dynamic models

(a) Using regression formulae

These parameters were obtained for regression equations as a part of a new technique of predicting the displacement of the suspension bridge taking into consideration the effect of several variables such as traffic load and meteorological factors (wind, temperature and humidity) as shown in Table 1.

Empirically the regression models for predicting derived mathematical equations showing the relation between the displacement in $\mathrm{cm}$ and some meteorological parameters (wind, temperature, humidity) and traffic load.

\section{(b) Using artificial neural network (ANNs)}

Second trial: Taking 50\% of all available data to analyze GNSS observations and build up the prediction model and the remaining $50 \%$ of data were used for checking (validating) that model with the confidence interval and probability as $\rho=0.95, \Delta= \pm 2 \sigma$ (Figs. 7 and 8 ).

Figures 9 and 10 show samples of the results of modeling GNSS observations in the $X$-direction for one receiver using $50 \%$ data of all available data in specified where the $X$-axis expresses the observations time in minutes and the $Y$-axis expresses the values of the $X$ coordinates component Fig. 11 and 12 .

(1) Solution using kinematic models

(2) For dynamic solution

(a) Using regression models

(b) Using ANNs
Fig. 3 Numbering of GNSS critical points of the studied suspension bridge

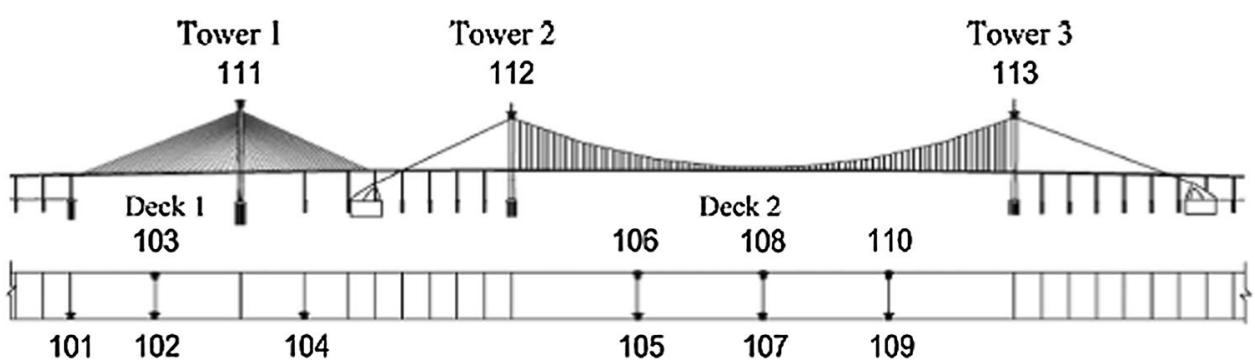




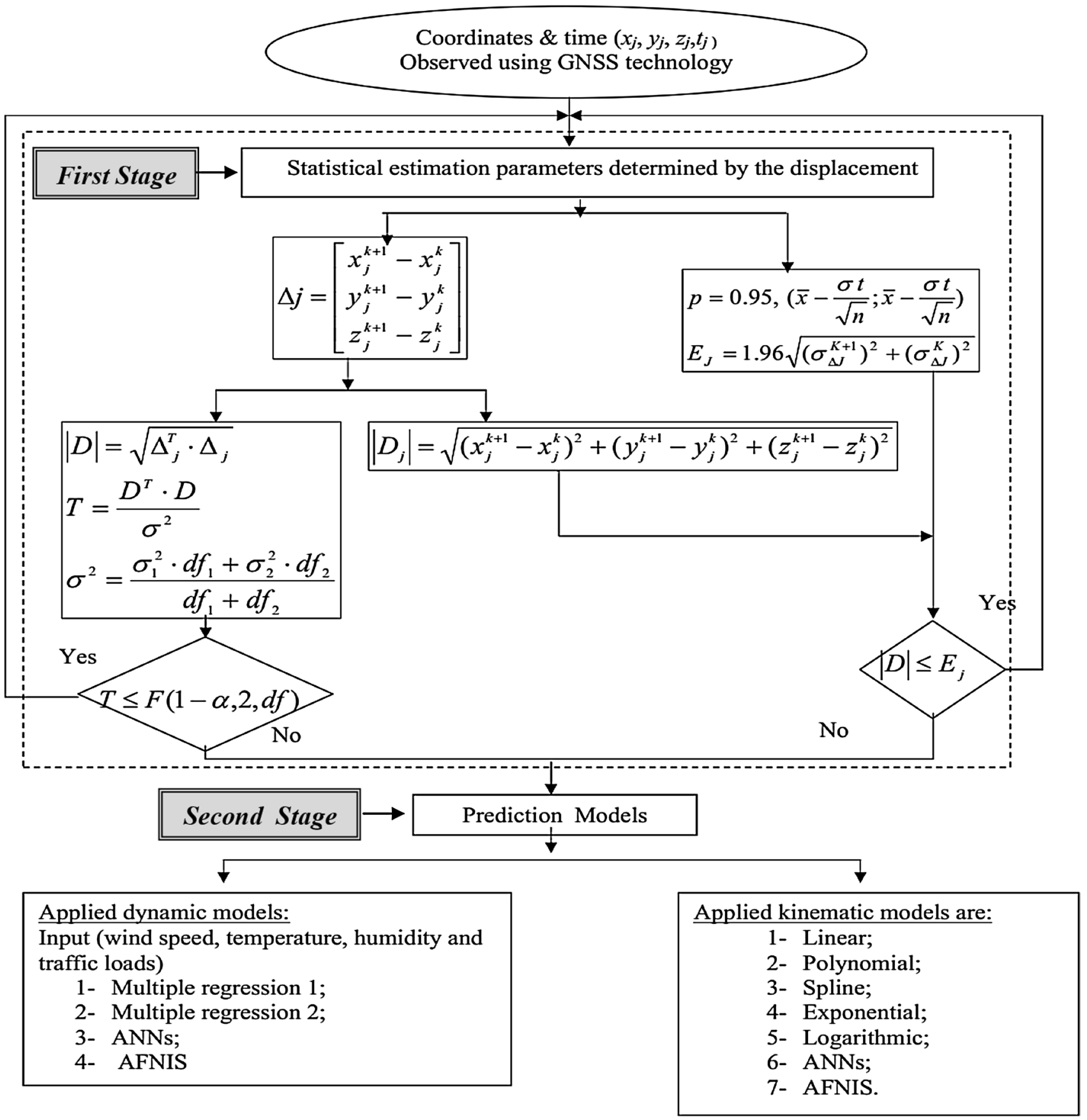

Fig. 4 Flowchart diagram explains the steps of applied solution for finding the prediction model

By comparing the results between the two trials (two different input data amounts for building the model) for two models (kinematic and dynamic) to compute the coefficients of the equations at the same condition (with the confidence interval with a probability $\rho=0.95, \Delta= \pm 2 \sigma$ ), it is deduced that ANNs model is better than regression models for processing GNSS observations and displacement prediction. Using $66.67 \%$ of data for building up the model is better than using $50 \%$ of all data because it improves the correlation coefficient by $5-10 \%$ when using artificial neural network and by $25-33 \%$ when using regression models.

\section{Third trial: using ANFIS for solution}

Two cases of amount data also are studied using adaptive neuro-fuzzy inference systems (ANFIS) for the two different solutions (kinematic and dynamic) as following: 


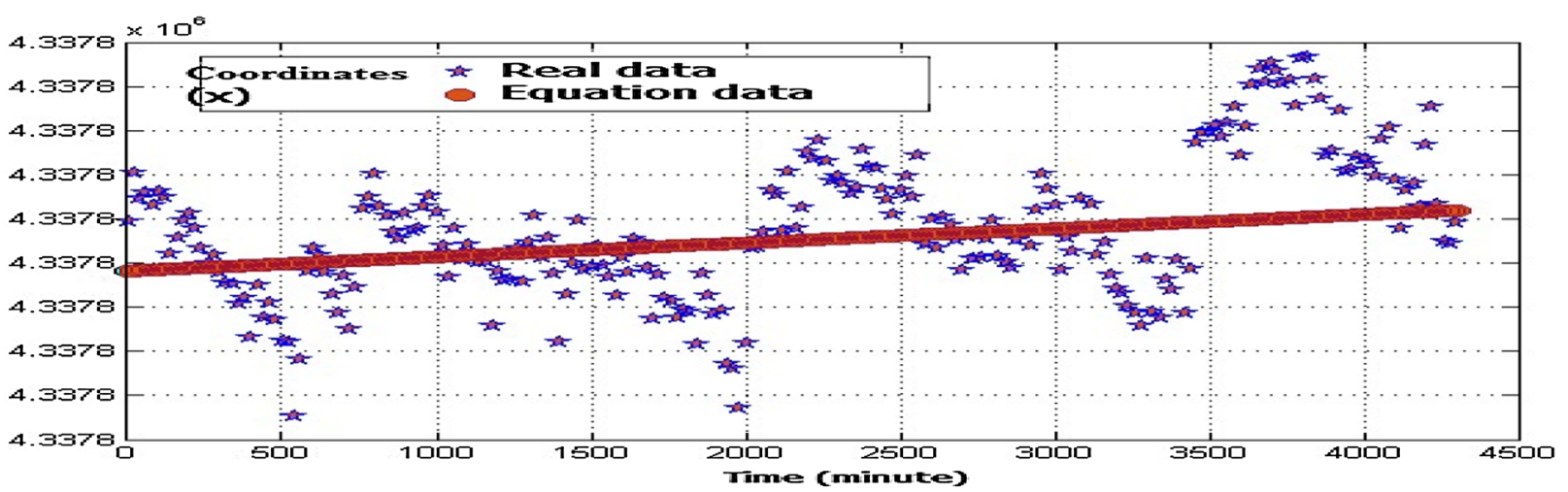

aLinear equation

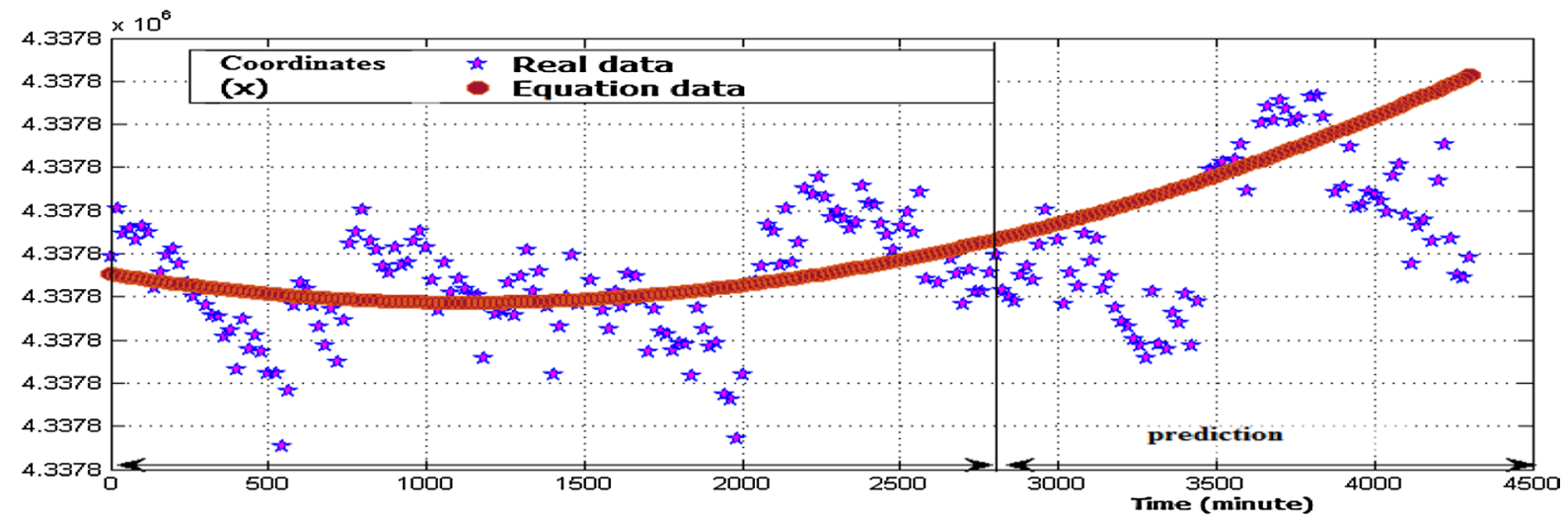

b Polynomial 2-nd degree

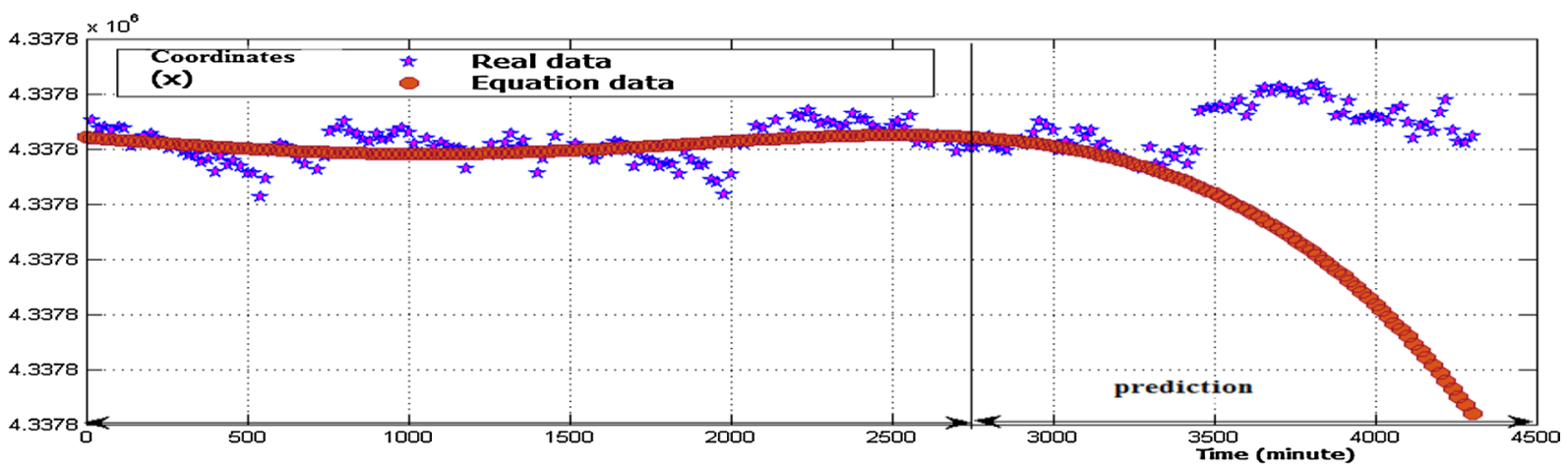

c Polynomial 4-th degree

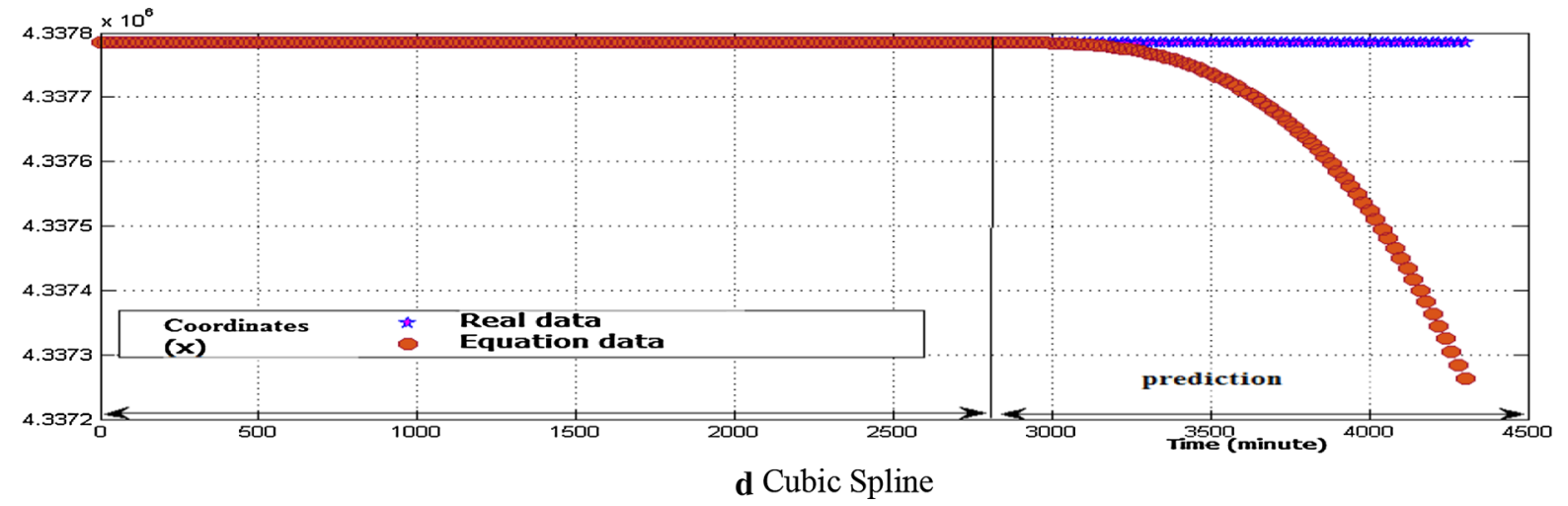

Fig. 5 The results of GNSS observations and prediction of $X$-displacement using kinematic models from regression analysis using $66.67 \%$ of all data 


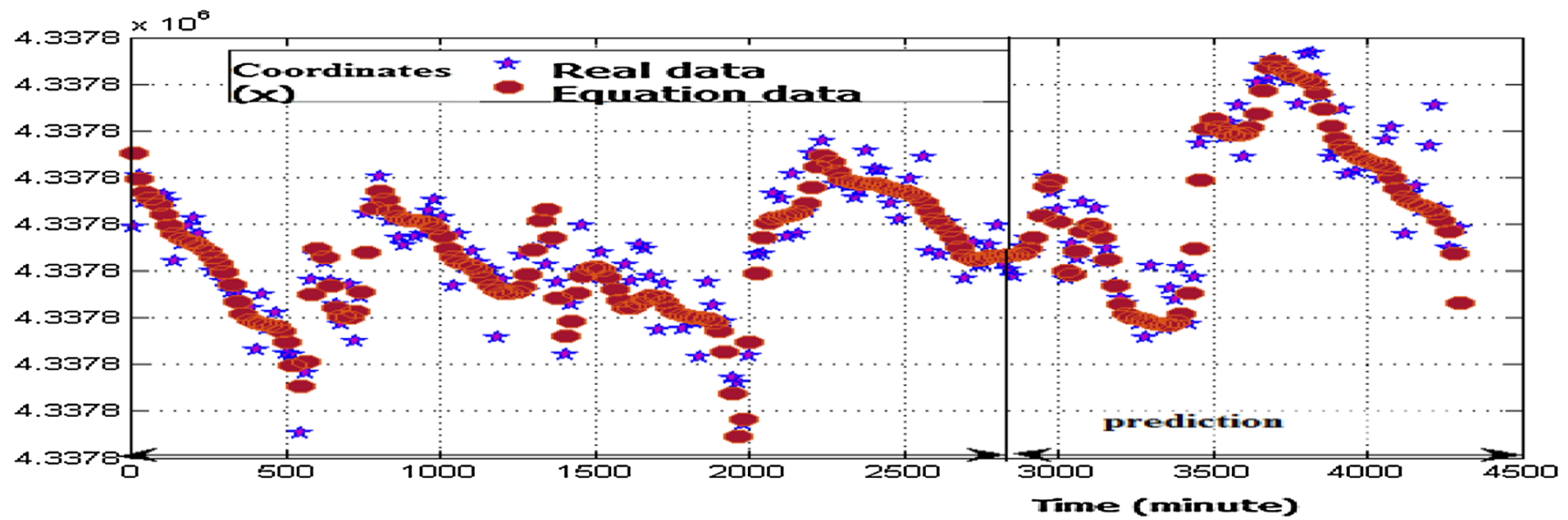

Fig. 6 The results of GNSS observations and prediction of $X$-displacements using kinematic models from artificial neural network (ANNs) using $66.67 \%$ of all data

Table 1 The coefficient of regression formulae for dynamic models using $66.67 \%$ of all available data

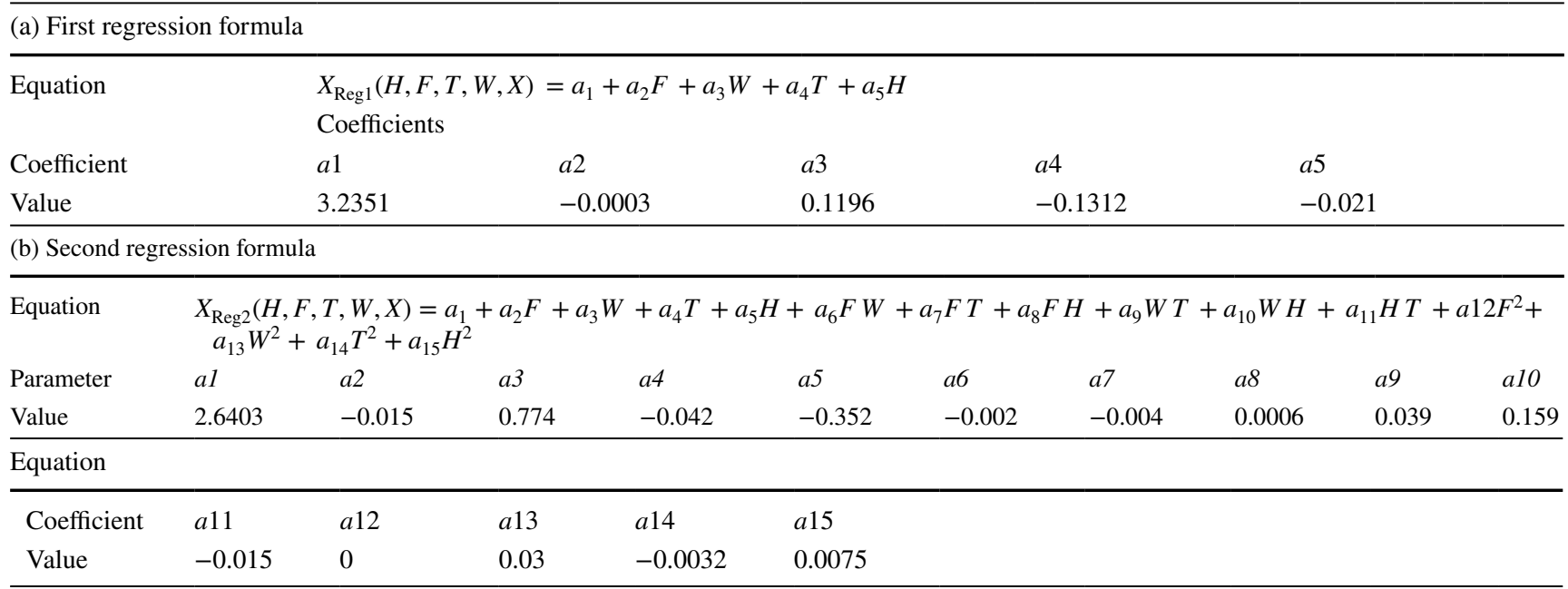

(1) For kinematic solution (displacement and time only)

(a) Taking $66.67 \%$ of all available data to analysis the GNSS observations and build up the prediction model and the remaining $33.33 \%$ of data for validation.

Figure 13 shows the results of modeling GNSS observations in the $Y$-direction (for example) for one receiver using $66.67 \%$ of data where the $X$-axis expresses the observations time in minutes and the $Y$-axis expresses the differences of $Y$ component coordinates value in $\mathrm{cm}$ (for example).

(b) Taking $50 \%$ of all available data to analysis the GNSS observations and build up the prediction model and the remaining $50 \%$ of data were used for checking (validating) that model.
The following figures show the results of modeling GNSS observations in the $Y$-direction for one receiver using $50 \%$ of data where the $X$-axis expresses the observations time in minutes and the $Y$-axis expresses the differences of the $Y$ component coordinates value in $\mathrm{cm}$.

(2) For dynamic solution

(a) Taking $66.67 \%$ of all available data to analysis the GNSS observations and build up the prediction model and the remaining $33.33 \%$ of data for validation.

The following figures show the results of modeling GNSS observations in the $Y$-direction for one receiver using $66.67 \%$ of data where the $X$-axis 




a Regression formula (1)

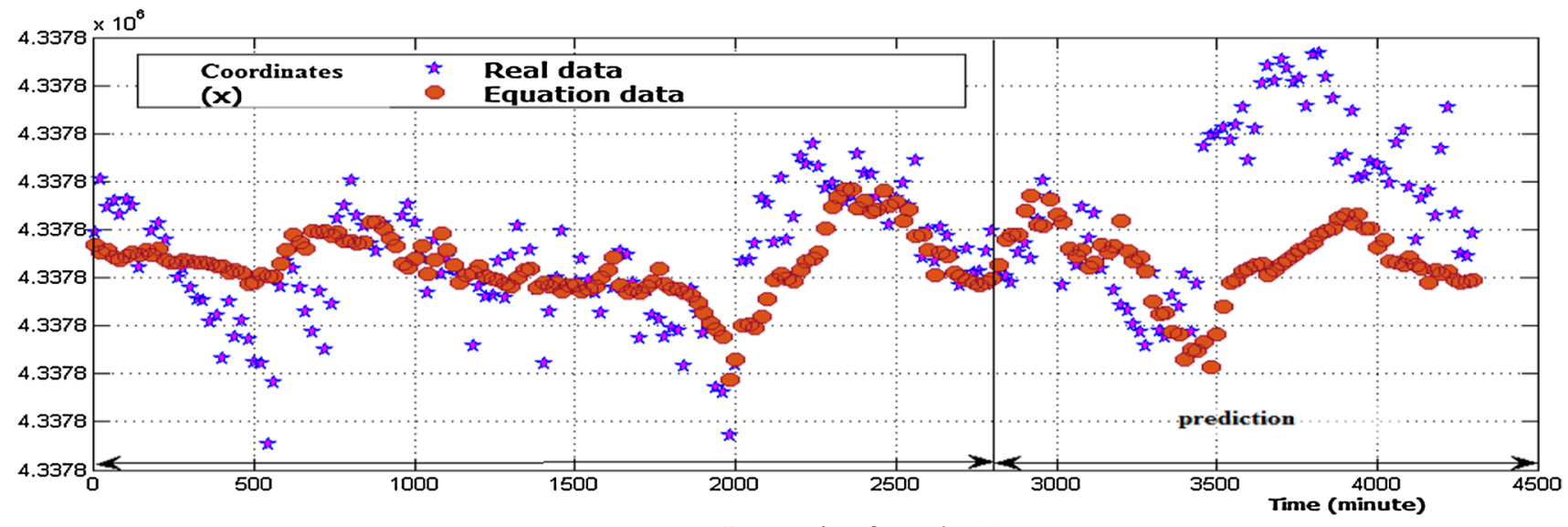

b Regression formula (2)

Fig. 7 The results of prediction for $X$-displacements using regression formula models using $66.67 \%$ of all data

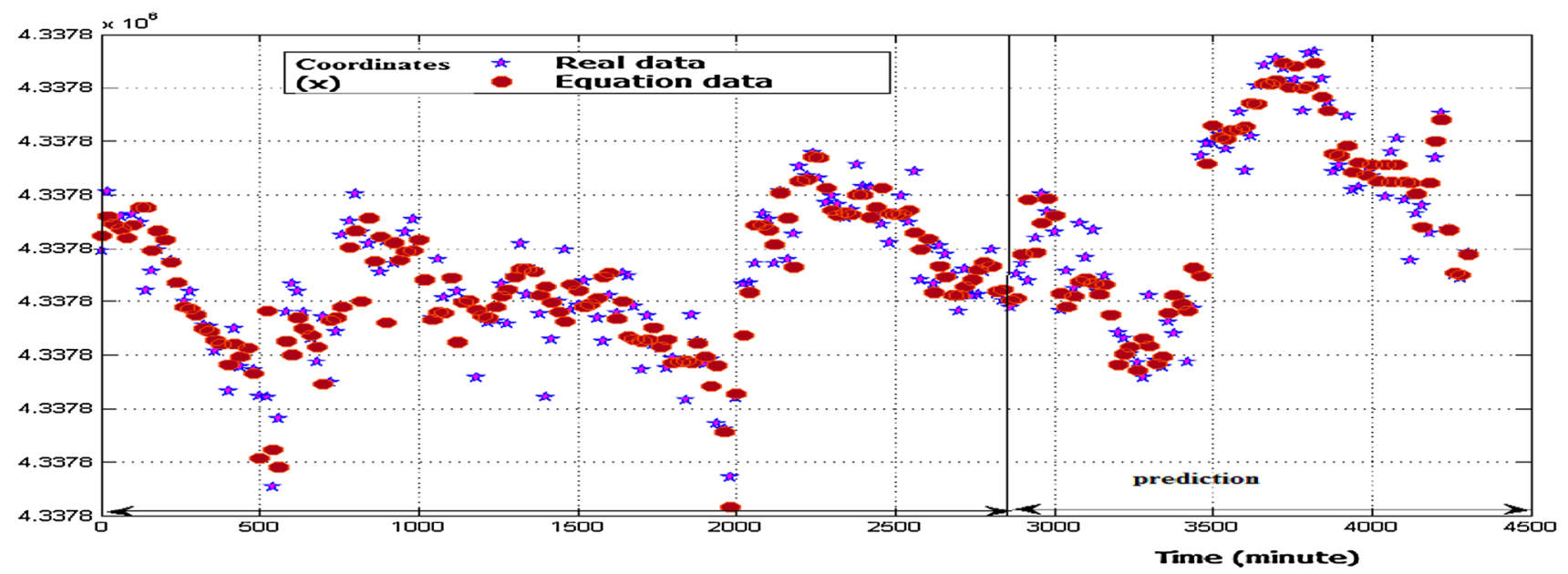

Fig. 8 The results of GNSS observations and prediction of $X$-displacements using dynamic model from artificial neural network (ANNs) using $66.67 \%$ of all data

expresses the observations time in minutes and the $Y$-axis expresses the differences of the $Y$ component displacements value in $\mathrm{cm}$. (b) Taking $50 \%$ of all available data to analysis the GNSS observations and build up the prediction 


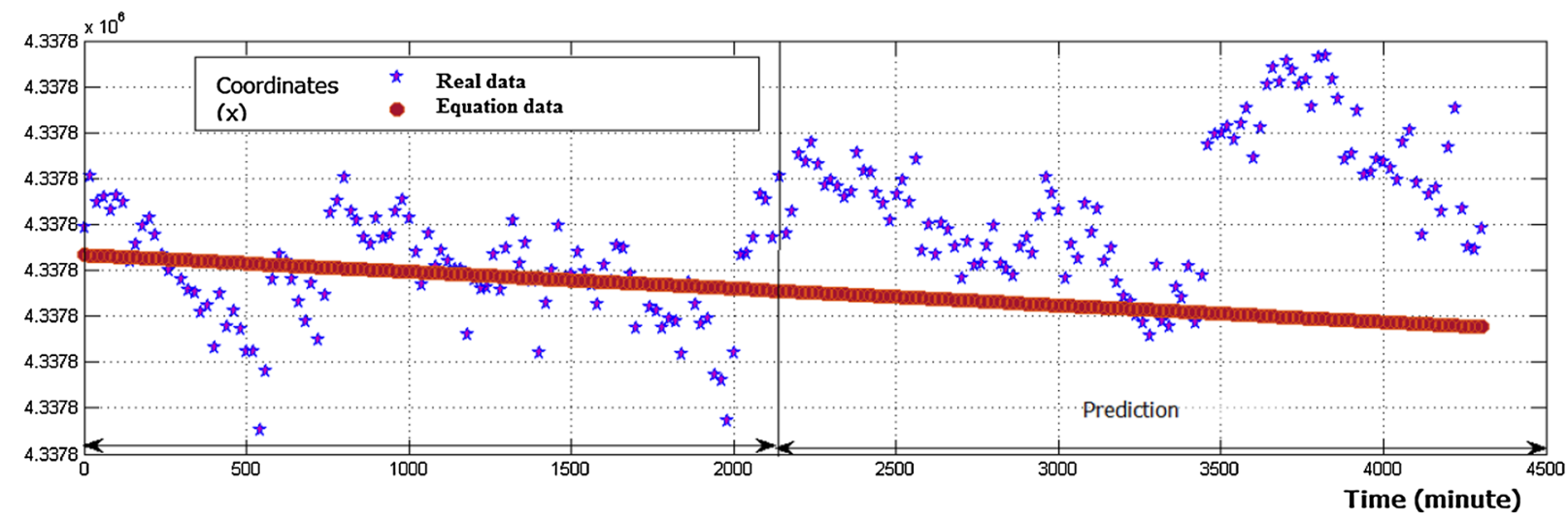

a Linear equation

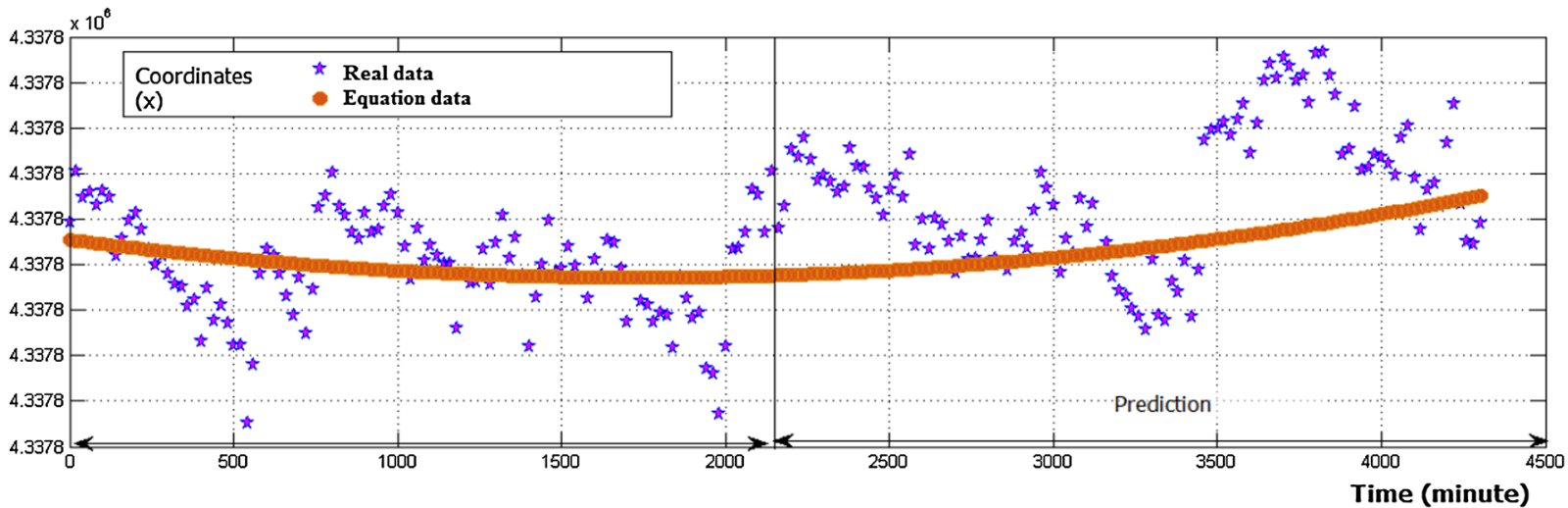

b Polynomial 2-nd degree

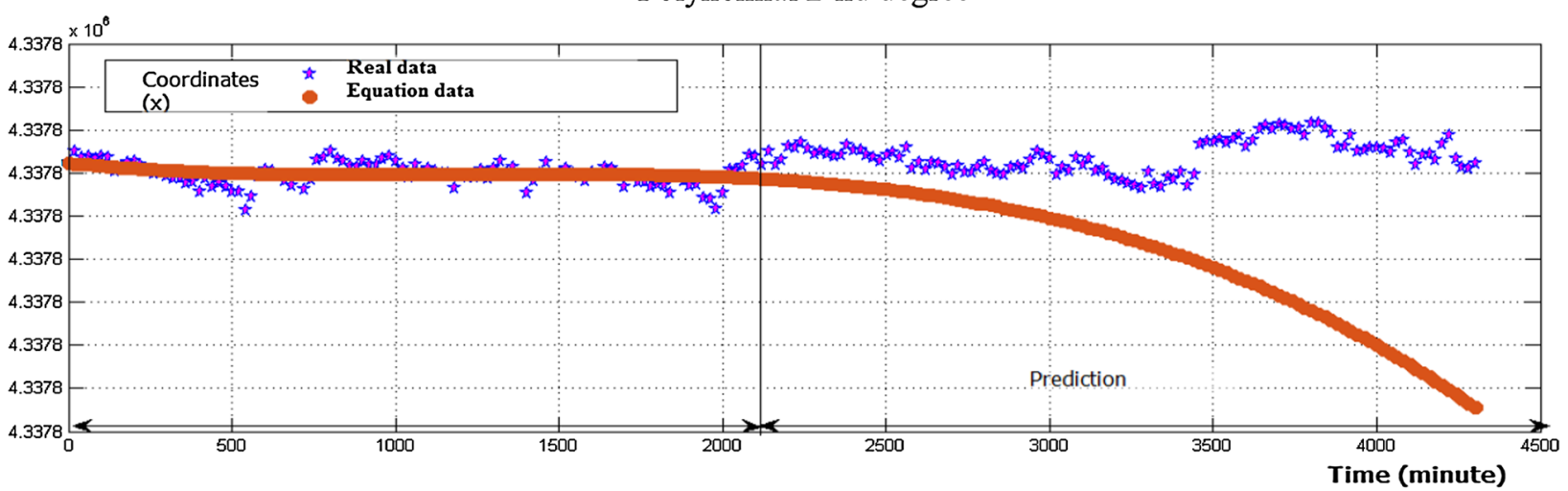

c Polynomial 4-th degree

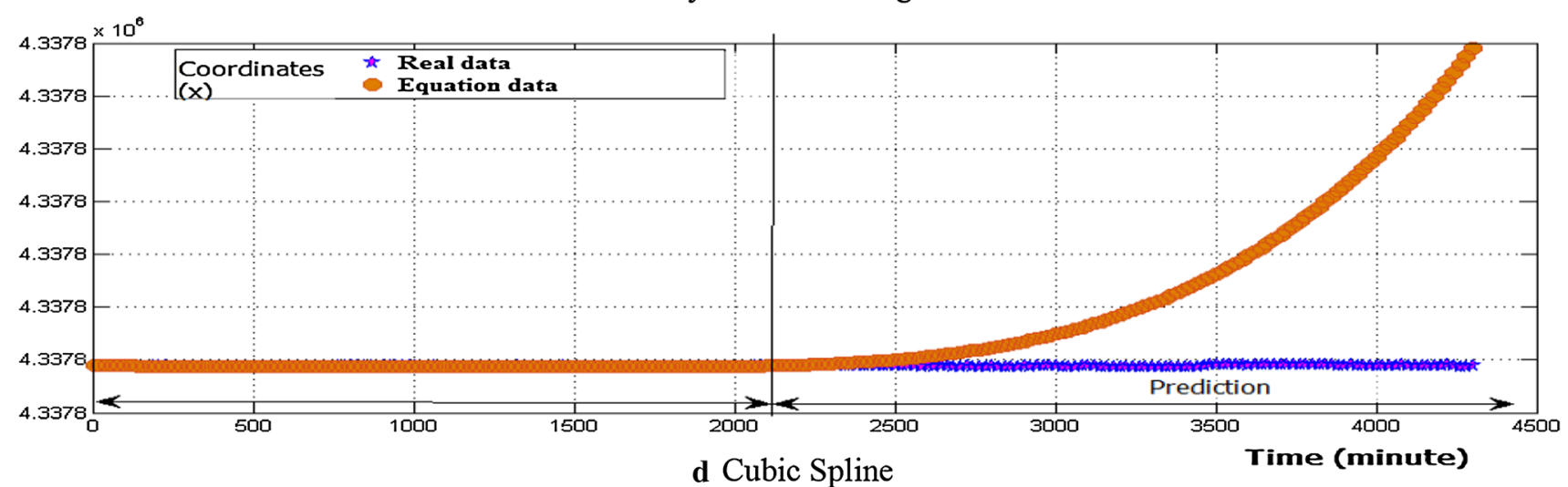

Fig. 9 The results of GNSS observations and prediction of $X$-displacement using kinematic models from regression analysis using $50 \%$ of all data 


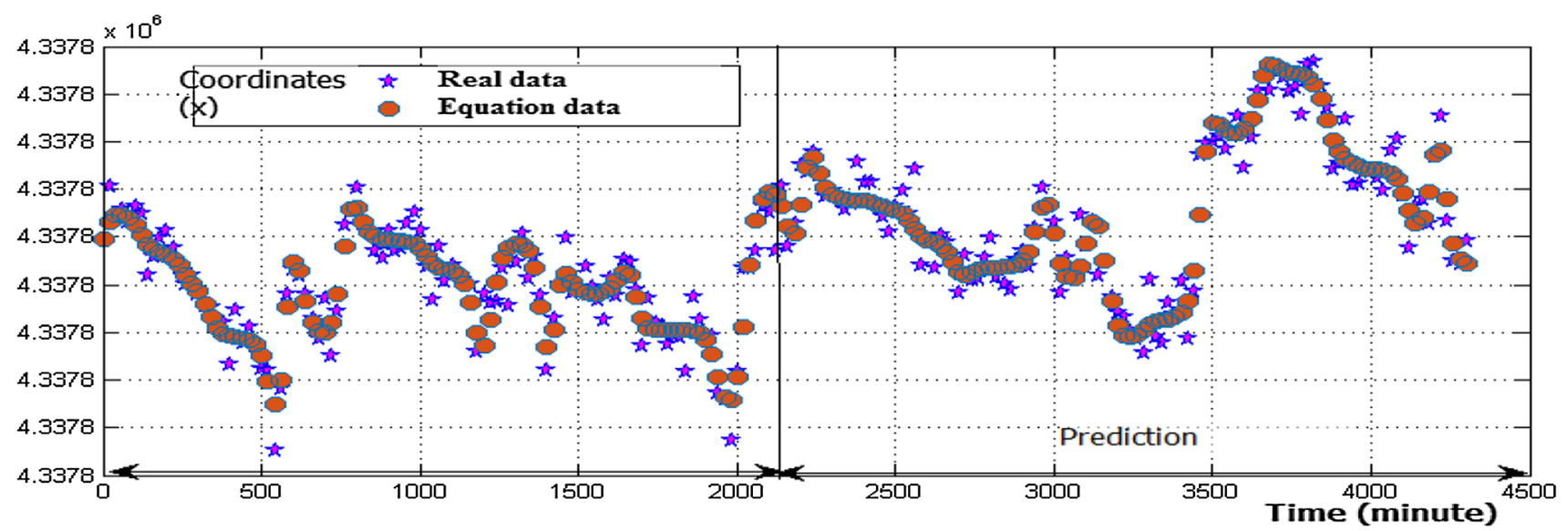

Fig. 10 The results of GNSS observations and prediction of $X$-displacements using kinematic models from artificial neural network (ANNs) using $50 \%$ of data

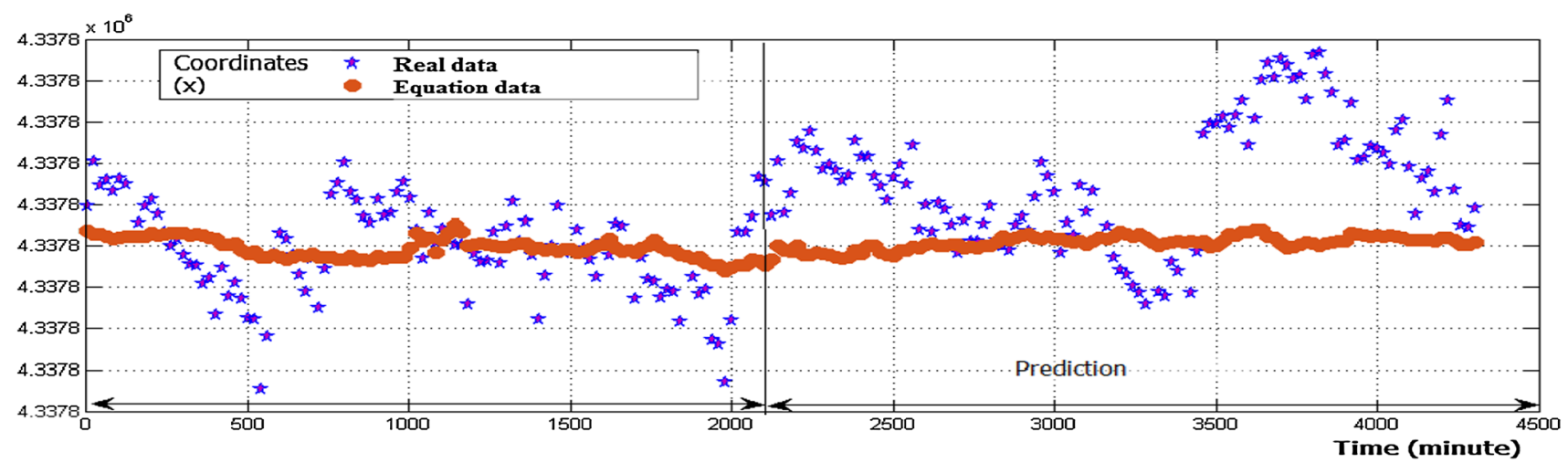

a Regression formula (1)

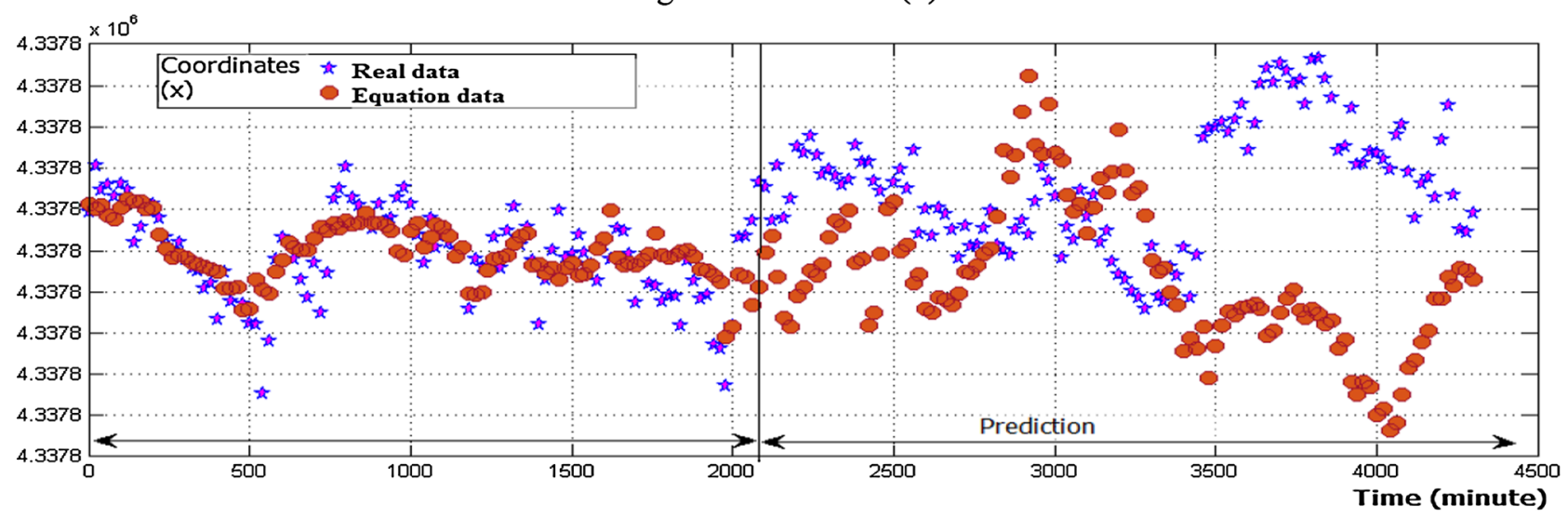

b Regression formula (2)

Fig. 11 The results of prediction for $X$-displacements using regression formula models

model and the remaining $50 \%$ of data were used for checking (validating) that model.
From Figs. 13, 14, 15 and 16, it is deduced that the root mean square error is improved by amount $14 \%$ when using $66.67 \%$ of all data than using $50 \%$ of all data for kinematic solution using adaptive neuro-fuzzy inference systems 


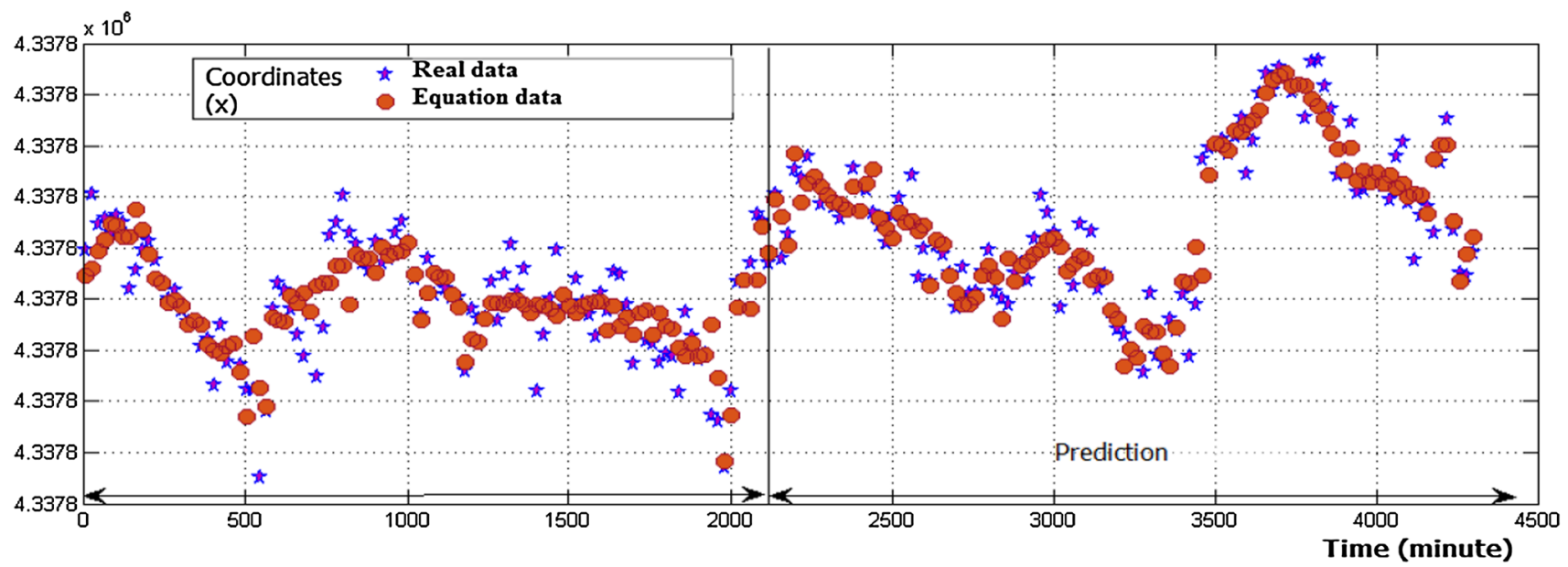

Fig. 12 The results of GNSS observations and prediction of $X$-displacements using dynamic model from artificial neural network (ANNs) using $50 \%$ of data
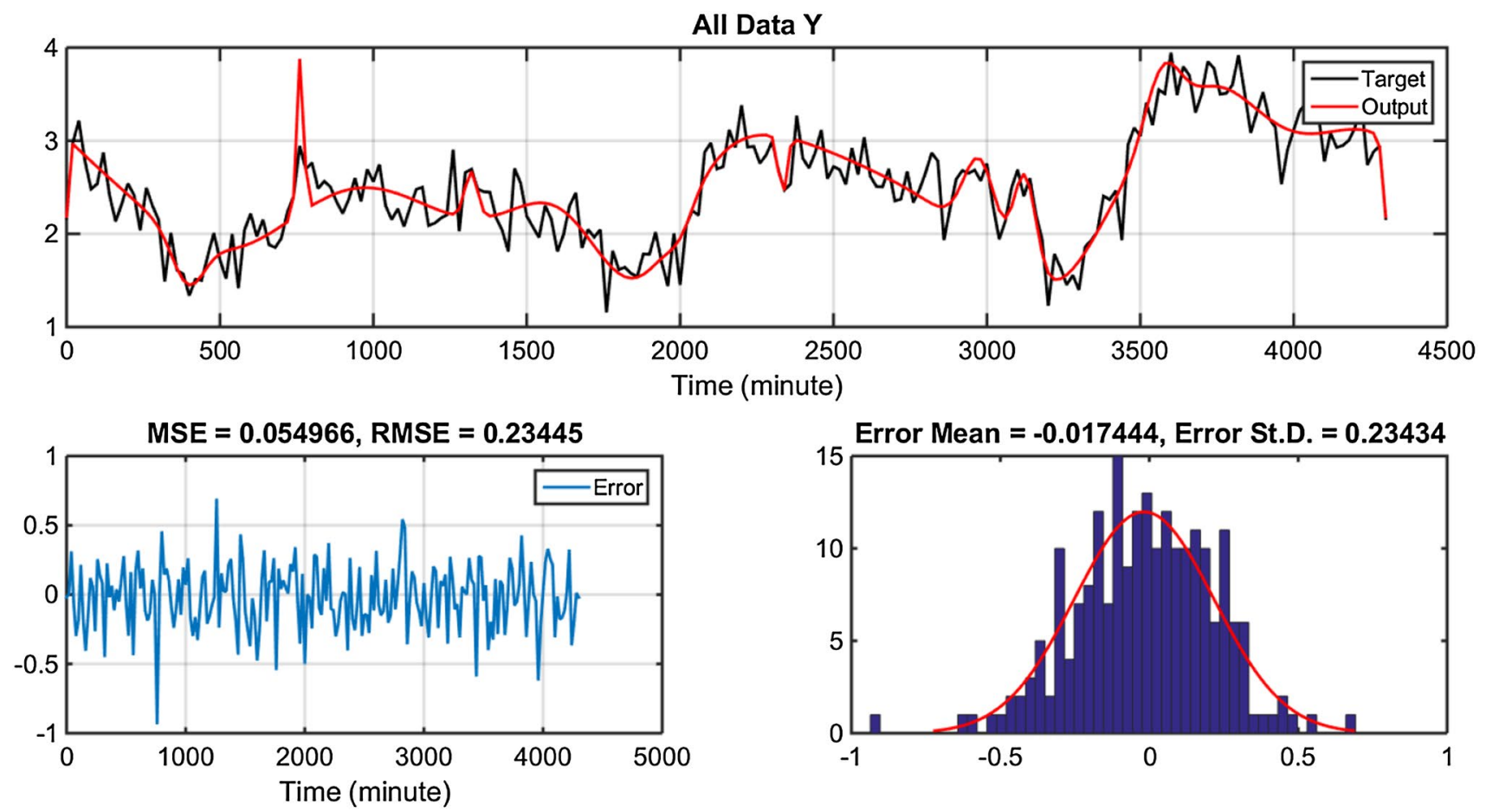

Fig. 13 The results of prediction for $Y$-displacements using evolutionary ANFIS using only $66.67 \%$ of data for kinematic model

(ANFIS) but it is improved by $60 \%$ for dynamic solution. This means that the amount of data has a great effect on the applied prediction model, therefore it is recommeneded that when building a satisfactory prediction model using GNSS observations, it should be use a large amount of data at different day times and at different days.

To compare all techniques applied in this paper for processing GNSS observation and find out the optimum displacement prediction model, the mean values of correlation coefficient for all monitoring points for all applied models are presented in Table 2 .

From the results arrived in Table 2, it is clear that (ANNs) and (ANFIS) get the best values for correlation coefficient rather than any other models. 

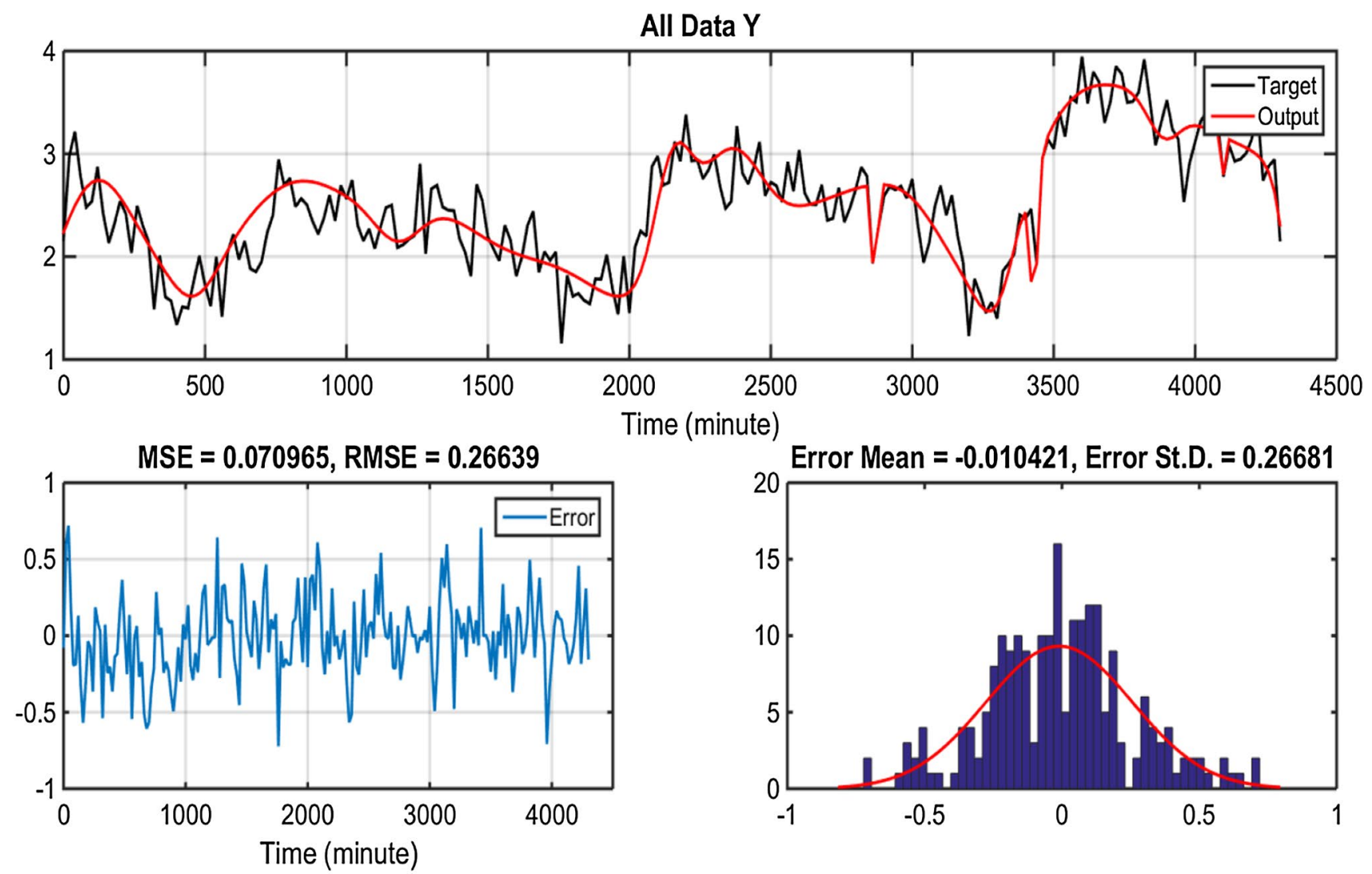

Fig. 14 The results of prediction for $Y$-displacements using evolutionary ANFIS using only $50 \%$ of data for kinematic model
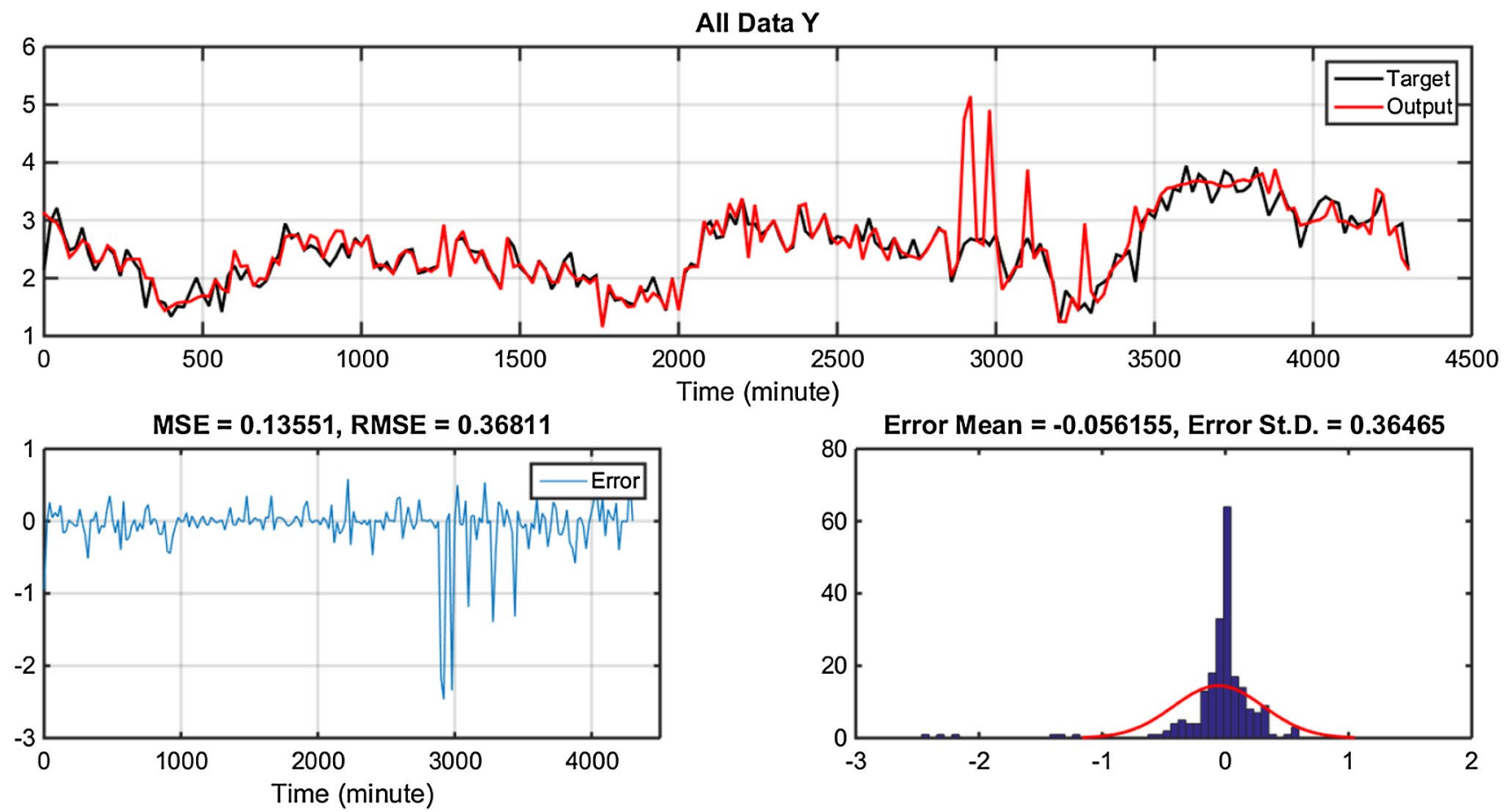

Fig. 15 The results of prediction for $Y$-displacements using evolutionary ANFIS using only $66.67 \%$ of data for kinematic model 

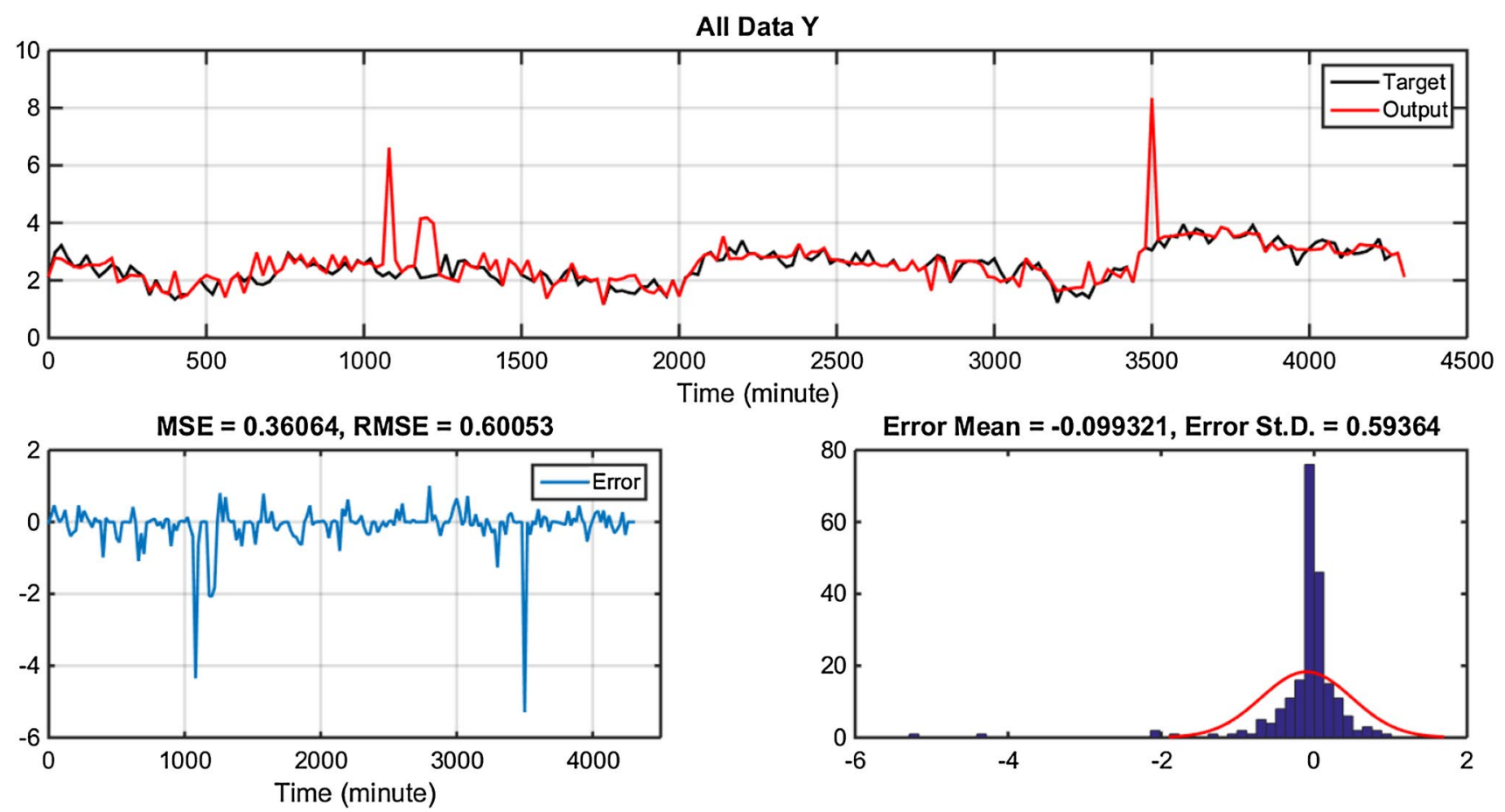

Fig. 16 The results of prediction for $Y$-displacements using evolutionary ANFIS using only $50 \%$ of data for kinematic model

Table 2 Correlation coefficients for all applied models and cases for all available data

\begin{tabular}{llll}
\hline Model & Function & Correlation coefficient $(R)$ & \\
\cline { 3 - 4 } & & $\begin{array}{l}\text { Using 66.67\% of all data for } \\
\text { building up prediction model }\end{array}$ & $\begin{array}{l}\text { Using 50\% of all data for } \\
\text { building up prediction } \\
\text { model }\end{array}$ \\
\hline Kinematic models & Linear & 0.221 & 0.203 \\
& Logarithmic & 0.235 & 0.195 \\
& Inverse & 0.197 & 0.184 \\
& Quadratic & 0.281 & 0.221 \\
& Cubic & 0.254 & 0.218 \\
& Power & 0.211 & 0.190 \\
& Compound & 0.206 & 0.161 \\
& Growth & 0.198 & 0.172 \\
& Exponential & 0.342 & 0.306 \\
& ANNs & 0.921 & 0.905 \\
& ANFIS & 0.954 & 0.93 \\
Dynamic models & Regression formula 1 & 0.372 & 0.311 \\
& Regression formula 2 & 0.391 & 0.328 \\
ANNs & 0.942 & 0.911 \\
ANFIS & 0.961 & 0.946 \\
\hline
\end{tabular}

\section{Conclusion}

Based on the analysis of results, the following conclusions can be drawn:
(1) The proposed geodetic automatic monitoring technique of the studied suspension bridge using GNSS observations technology can provide a large amount of information and valuable data every few seconds, which 
help to understand and evaluate the health and safety of that structure, and therefore makes the assessment of their security more reliable.

(2) There are several mathematical models and techniques which can be used to process GNSS observations of suspension bridges as presented. These models are used to predict the deformation and morphological values of bridge structural elements in kinematic and dynamic cases. The resulting predicted displacement values by applying ANNs and ANFIS, which used a confidence interval with a probability of $\rho=0.95, \Delta= \pm 2 \sigma$, are more accurate and reliable than any other applied mathematical methods, therefore ANNs and ANFIS can provide an improvement of understanding and predicting the structure deformation values.

(3) The applied models and techniques for GNSS observations processing and displacement prediction for suspension bridges depend mainly on the amount of data observed and confidence limit. In this paper, using $66.67 \%$ of all available data for building up the model is better than using $50 \%$ of data because it improves the correlation between the observations (original data) and the resulting observations of the model by $5-10 \%$ when using artificial neural network (ANNs and ANFIS) with a confidence interval with a probability of $\rho=0.95$, $\Delta= \pm 2 \sigma$ and by $25-33 \%$ when using regression models.

(4) Adaptive neuro-fuzzy inference systems (ANFIS) can be considered as the optimum model and technique for GNSS observations processing and displacement prediction for suspension bridge or any other structure for kinematic (displacement and time only) and dynamic state [displacement and other factors (vehicle load, wind, temperature, humidity, time)].

Acknowledgements The authors want to thank Dr. Mosbeh Rashed Mosbeh, Faculty of Engineering, Mansoura University for providing the data (observations) of the studied suspension bridge.

\section{Compliance with ethical standards}

Conflict of interest On behalf of all authors, the corresponding author states that there is no conflict of interest.

Open Access This article is licensed under a Creative Commons Attribution 4.0 International License, which permits use, sharing, adaptation, distribution and reproduction in any medium or format, as long as you give appropriate credit to the original author(s) and the source, provide a link to the Creative Commons licence, and indicate if changes were made. The images or other third party material in this article are included in the article's Creative Commons licence, unless indicated otherwise in a credit line to the material. If material is not included in the article's Creative Commons licence and your intended use is not permitted by statutory regulation or exceeds the permitted use, you will need to obtain permission directly from the copyright holder. To view a copy of this licence, visit http://creativecommons.org/licenses/by/4.0/.

\section{References}

1. Kaloop MR, Hussana M, Kima D (2019) Time-series analysis of GPS measurements for long-span bridge movements using wavelet and model prediction techniques. J Adv Space Res 63(11):3505-3521

2. USACE (2002) Structural deformation surveying (EM 1110-21009). US Army Corps of Engineers, Washington, DC

3. Beshr AAA (2010) Development and innovation of technologies for deformation monitoring of engineering structures using highly accurate modern surveying techniques and instruments. Ph.D. thesis, Siberian State Academy of geodesy SSGA, Novosibirsk, Russia, p 205

4. Vanatwerp RL (1994) Engineering and design: deformation monitoring and control surveying, Engineer manual. EM 1110-1-1004. US Army corps of engineering, Washington US, $p 141$

5. Kaloop MR, Li H (2009) Monitoring of bridges deformation using GPS technique. KSCE J Civil Eng (KSCE) 13:423-431

6. Zarzoura F, Mazurov B, Ahmed C (2015) Geodetic monitoring cable-stayed bridges using GNSS. FIG working week 2015, from the wisdom of the ages to the challenges of the modern world, Sofia, Bulgaria, 17-21 May 2015, ID No 7717, p 10

7. Cankut DI, Muhammed S (2000) Real-time deformation monitoring with GPS and Kalman filter. Earth Planets Space 52(10):837-840

8. Akyilmaz O, Celik RN, Apaydin N, Ayan T (2004) GPS monitoring of the Fatih Sultan Mehmet suspension bridge by using assessment methods of neural networks. Int Arch Photogramm Remote Sens Spat Inf Sci 34:702-707

9. Krishna MSV, Begum KMS, Anantharaman N (2017) Hydrodynamic studies in fluidized bed with internals and modeling using ANN and ANFIS. Powder Technol 307:37-45

10. Keshavarz Z (2018) Application of ANN and ANFIS models in determining compressive strength of concrete. J Soft Comput Civ Eng 2-1:62-70. https://doi.org/10.22115/SCCE.2018.51114

11. Beshr AAA (2012) Monitoring the structural deformation of tanks. LAP LAMBERT Academic publishing, Germany, p 284

12. Azar RS, Shafri HZ (2009) Mass structure deformation monitoring using low cost differential global positioning system device. J Appl Sci, ASCE 6(1):152-156

13. Mellit A, Saglam S, Kalogirou SA (2013) Artificial neural network based model for estimating the produced power of a photovoltaic module. Renew Energy 60:71-78

14. Haykin S (2001) Kalman filtering and neural networks. McMaster University, Communication Research Laboratory, Hamilton, Ontario, Canada

15. Wieland D, Wotawa F, Wotawa G (2002) From neural networks to qualitative models in environmental engineering. Comput Aided Civ Infrastruct Eng 17:104-118. https://doi.org/10.1016/j. asr.2019.02.027

16. Jang JSR (1993) ANFIS: adaptive-network-based fuzzy inference system. IEEE Trans Syst Man Cybern 23:665-685 